https://doi.org/10.48009/2_iis_2009_466-471

\title{
THE BUSINESS TRADEOFFS WITH THE FINAL - FOUR MODELS OF CONVENTIONAL TELEPHONY SYSTEMS
}

\author{
Kelly, Timothy V. Elizabeth City State University, tvkelly@ mail.ecsu.edu
}

\begin{abstract}
Several factors become apparent when a business is compelled to evaluate the decision to go with VoIP and a unified communications model or continue with one of the four conventional telephony systems model solutions. VoIP brings with it high start-up costs typically coupled with a multi- month payback period. However, these costs are followed by an ongoing total cost of operation (TCO) savings. These costs and savings impact the operations of the computer network which now would include, data; and operation of telephony and video-conferencing systems. VoIP supports unified communications across the enterprise. The purpose of this exploratory research is to examine the system tradeoffs and the cost differences among the Final-Four Models of conventional telephony systems in relation to converting to VoIP.
\end{abstract}

Keywords: VoIP, Telephony Information Systems, CENTREX, PBX, IP-PBX, Telephony Regulations

\section{INTRODUCTION}

Despite the global economic downturn over the last two years, the number of corporate telephony systems provider companies in the U.S. has grown. Many of these provider companies have "voice over internet protocol" (VoIP) design packages - among the top of their core product/service business lines. Since VoIP became marketable, the four traditional telephony system models have been rendered noncompetitive. Converged, integrated communications, deploying VoIP technologies has arrived [2;3].

Before VoIP matured, companies were compelled to use one of the four standard telephony systems (See Table 2) to operate their business. These conventional telephony systems were physically separate from the company's computer network infrastructure. If video- conferencing was needed, the company had to install a third infrastructure. VoIP enables a "unified" architecture that integrates telephony, computer and video-conferencing systems into a single network $[1 ; 6]$.

\section{UNIFIED COMMUNICATIONS}

Today more companies continue to operate with separate telephony, computer and video network infrastructures, compared to companies that have converted to VoIP. At the same time, more companies than ever before are looking for alternatives to integrate their separated networks into a single unified infrastructure. VoIP optimizes unified communications $[2 ; 6]$.

"Consolidation in the enterprise telephony market continues as the decisions for investing in UC takes precedence over telephony" [2]. Although, companies are still deploying Premises Business Exchange (PBX) types of telephony systems (See Table 2). A PBX is not only one of the final four models; it is the most expensive of the four. At the same time, there are more positive tradeoffs for a business that runs with a PBX telephony system today versus any of the other three models. For example, with a PBX system a company can run VoIP. Therefore, a PBX system can be made to be the centerpiece of a company's computer and videoconferencing networks. In this way, computer data, telephony systems and videoconferencing interoperate over the same, single infrastructure. Recurring telephony access and usage costs are eliminated from the company's total cost of operation (TCO) $[1 ; 7 ; 9]$.

\section{COST TRADEOFFS}

Needless to say, the cost tradeoffs became obvious when VoIP systems began replacing conventional telephony systems in the corporate sector. Instead of two or three separate infrastructures to support all of their information systems, companies could design their Enterprise IT System to operate on one single infrastructure $[1 ; 2 ; 5]$. That infrastructure was enabled by VoIP. Moreover, there were intrinsic cost-savings from the new VoIP-based infrastructure.

Though VoIP conversions may often come with typically high startup costs, the resulting corporate change over to an Enterprise IT System based in VoIP, brought about eventual savings, or complete elimination of recurring costs associated with all four of the conventional telephony system models. 


\section{COST SAVINGS}

Much of the savings from converting to VoIP and replacing or upgrading older telephony systems to support VoIP, come about by reducing or eliminating recurring per-call carrier service charges. However, companies making the change over to VoIP also save the monthly recurring charges (MRC) associated with the "calling category" regulatory costs. Average monthly telephone call regulatory costs add as much as $4 \%-7 \%$ to the company's total MRC in non-VoIP businesses that still operate with the "switched" telephony system models. These regulatory costs are based on five regulated calling-categories. Table 1 illustrates these five categories $[2 ; 5 ; 6 ; 8]$.

\section{REGULATORY COSTS}

Regulatory charges stemming from fees, surcharges and taxes are typically eradicated or greatly reduced for companies operating under VoIP. The category of "International" regulatory costs is by far the most highly regulated of the five telephony categories. In a private, multi-national corporate network, running VoIP across the enterprise, all calls made on the corporate network have no regulatory costs and no carrier service charges $[1 ; 2 ; 5 ; 6]$.

Table 1 The Five Regulated Calling Categories of Switched Telephony

\begin{tabular}{|l|l|l|}
\hline $\begin{array}{l}\text { Regulated } \\
\text { Calling } \\
\text { Category }\end{array}$ & $\begin{array}{l}\text { Degree of } \\
\text { Regulatory } \\
\text { Costs }\end{array}$ & $\begin{array}{l}\text { Degree of } \\
\text { Regulatory Cost } \\
\text { Reduction With } \\
\text { VoIP }\end{array}$ \\
\hline Local & least & least \\
\hline Intralata & moderate & most \\
\hline Intrastate & moderate & high \\
\hline Interstate & high & moderate \\
\hline International & most & high \\
\hline
\end{tabular}

For example, a telephone could be dialed from a VoIP telephone in the Pittsburgh office of a Fortune 500 steel manufacturer to a destination telephone number of a telephone located in a subsidiary's office in London. The call would be packetized using the caller's Internet Protocol (IP) address, User Datagram Protocol (UDP) and Network Transmission Protocol (NTP) on the Pittsburgh office network.

The call is then forwarded on electronically to the company's regional network in packet form. The network's router inserts the destination IP address (which correlates to the telephone number of the person being called). The router also completes the outgoing packets with the other protocols needed.
The packetized call is then propagated on to the company's global network with a destination address of the London office's telephone. When the London telephone first rings, a virtual circuit is then established for the life of the call over the company's private network.

At no time in the above example did any component parts or packets of the telephone call touch, crossover, transmit or otherwise get "carried" on a regulated network. Therefore, there are no carrier service charges and no regulatory costs, including those from the international category. (Note: that with international calls over regulated networks, each of the two countries, on either side of the call will assess the fees, surcharges and taxes to the party initiating the call.

\section{NON-VOIP SYSTEM TRADEOFFS}

In a non-VoIP enterprise, the call would have been dialed, initiated, carried over and maintained on the Public Switched Telephone Network (PSTN). Multiple regulated networks would have been discretely involved to support the life of the call. As a result, the customer-company would pay the perminute recurring charges. In addition, the company would also have to pay the International regulatory fees, surcharges and taxes from both sides of this call, that is, the U.S. (Pittsburgh) side as well as the U.K. (London) side [8].

When considering the non-VoIP system tradeoffs take into account the reduction or elimination of the total charges relating to carrier-services. These include recurring per-minute usage charges and the added regulatory costs for all lines in the non-VoIP network. Using this type of analysis, the case can usually be made, based on cost alone, that it is in the long-term interest of the company to plan for conversion of their enterprise network to operate under VoIP.

In addition to the cost savings, VoIP networks provide several other features and applications which would have to be "add on" costs for a non-VoIP company network.

\section{ADD-ON SYSTEM FEATURE COSTS}

VoIP networks include the capability to seamlessly add video-conferencing to some or all of the company's network locations. In a non-VoIP network, the company would need to build a third network infrastructure to support video-conferencing in this manner. Also, traditional telephone features are integrated into the VoIP telephony network system. These features, to list a few out of the many 
that come with VoIP at no extra cost, include: caller identification, VEMAIL (email and voice-mail combined), conference calling and many more [10].

These features are identified here because when considering the tradeoffs between the Final Four Models of telephony systems, it is important to know that to obtain these features under a VoIP Model there is no additional cost. Indeed features are considered billable add-ons in the POTS and CENTREX Models of the Final-Four Models. Each feature will have an individual MRC cost attached to it. Table 2 summarizes the Final Four Models of conventional telephony systems.

\section{A "POTS LINE SYSTEM" MODEL EXAMPLE}

What determines the extent of VoIP startup conversion costs is based on which of the four conventional telephony systems are in place at the time of conversion. If the company is operating with a system of individual plain old telephone (AKA: POTS) lines, it will come down to how many total POTS lines, what calling-pattern types the lines are used for (see Table 1) and how many minutes per month the lines are used in this manner.

Conversion costs can be offset by VoIP savings. To calculate overall cost or how much can be saved; the conventional telephony system's recurring costs need to be determined. The determination is based on the total number of POTS lines, the total number of minutes per month that each POTS line is used for and the total number of minutes per line subdivided by the types of calls made as illustrated in Table 1 with the five distinct regulated call categories.

For example, intralata calls (sometimes called "extended local" or "regional" calls) made in the northeast region of the U.S., range in recurring cost from $\$ 0.12$ cents per minute to $\$ 0.64$ cents per minute, making the average cost per minute equal to $\$ 0.38$ cents per minute. If the company has two hundred POTS lines, in total, across their enterprise organization and they expend in an average month approximately 50,000 total minutes of intralata calls, their approximate cost just for their intralata calling patterns, and not including fees, surcharges or taxes is $\$ 19,000$.

A VoIP enterprise in the above example would eliminate the entire $\$ 19,000$ cost or at worst reduce each call to a "local" business call. Local business calls today average a flat charge of $\$ 0.05$ cents with no recurring minute charges. Since the average length of a business call is about three minutes, the total monthly billable minutes in this example reduces to approximately 16,700 calls per month.
If we multiply the $\$ 0.05$ cents by 16,700 calls, our worst case cost under VoIP, again not including fees, surcharges or taxes, would be approximately $\$ 875$. That is an approximate difference or savings of $\$ 18,000$ dollars. (These savings are just for what use to be the MRCs for intralata calls for this company.)

The same formula could be applied to calculate the savings under VoIP for the other regulated categories listed in Table 1. The regulatory costs will change. These non-VoIP costs are often referred to as "toll" charges. VoIP is often referred to as a technology that enables the customer to bypass these toll charges.

\section{TOLL BYPASS CHARGES}

The other conventional telephony system models improve on the monthly recurring cost factor and they reduce the need for the number of POTS lines linearly. However, the startup cost for these systems is also considered high and they do not come with the many advantages and features that a company operating with VoIP would have.

We could go through each calling category represented in Table 1 and calculate similar scenarios for a company that is considering making the conversion from their conventional system model to VoIP. It is highly likely we would find that in each category specified in Table 1 , there would be a savings at the end of each month.

However, the "toll bypass charges" apply differently to each of the five regulated categories. The term "toll bypass" is used because what we used to call long-distance charges or a telephone call which had "toll" charges has been divided up by the government into these five categories listed in Table 1. The only category that no longer has toll charges associated with it is the "Local" calling area.

\section{THE FINAL-FOUR MODELS}

The preexisting conventional telephony system that a company has in place needs to be thoroughly considered in terms of how it will play a role, if any, in the forthcoming conversion to VoIP. It is possible to run VoIP in varying degrees over any of the FinalFour Model telephony systems. The question becomes "Can you support an entire enterprise by running VoIP over a conventional telephony system?"

For example, in the Premises Business Exchange (PBX) Model of conventional telephony systems in 
Table 2 , it is possible to actually use the PBX system to support the conversion. Dedicated line (s) having far greater bandwidth can be used with a PBX, instead of the POTS lines. With dedicated lines the company can operate computer data, and both voice and video over Internet Protocol. Consequently, tradeoffs exist with the conventional PBX telephony model that enables a company to migrate to VoIP while still protecting their investment in preexisting telephony systems.

Prior to VoIP there were four models to guide a company in choosing its telephony information systems. These four models, all of which are still thriving in the current telecommunications and networking marketplace are summarized here in Table 2 by system name, equipment location and applicable cost structure.

Table 2 Final-Four Models of Conventional Telephony Systems

\begin{tabular}{|c|c|c|}
\hline $\begin{array}{l}\text { System } \\
\text { Name }\end{array}$ & $\begin{array}{l}\text { Location of } \\
\text { Equipment }\end{array}$ & $\begin{array}{l}\text { Applicable } \\
\text { Cost } \\
\text { Structure }\end{array}$ \\
\hline POTS & $\begin{array}{l}\text { Carrier lines run } \\
\text { to employer } \\
\text { owned } \\
\text { telephones. }\end{array}$ & $\begin{array}{l}\text { Monthly recurring } \\
\text { access and usage } \\
\text { charges per-line, per } \\
\text { phone. Regulatory } \\
\text { costs apply to total } \\
\text { cost of each call. }\end{array}$ \\
\hline KTS & $\begin{array}{c}\text { For every six- } \\
\text { eight employees, } \\
\text { one POTS carrier } \\
\text { line is run to } \\
\text { company's KTS. }\end{array}$ & $\begin{array}{c}\text { Same cost structure as } \\
\text { POTS based on each } \\
\text { line. Free call } \\
\text { features. Company } \\
\text { must buy/lease KTS. }\end{array}$ \\
\hline $\begin{array}{c}\text { CENT- } \\
\text { REX }\end{array}$ & $\begin{array}{l}\text { POTS equivalent } \\
\text { lines run from } \\
\text { Carrier's System } \\
\text { to all employees' } \\
\text { telephones. }\end{array}$ & $\begin{array}{l}\text { Higher line, call } \\
\text { feature and change } \\
\text { costs. Regulatory } \\
\text { costs apply to total } \\
\text { cost of each call. }\end{array}$ \\
\hline PBX & $\begin{array}{c}\text { Dedicated carrier } \\
\text { lines (i.e. T1, T3) } \\
\text { connected to the } \\
\text { company's PBX } \\
\text { System. }\end{array}$ & $\begin{array}{c}\text { Dedicated access } \\
\text { (AKA: Private line) } \\
\text { means no carrier usage } \\
\text { costs. Free call } \\
\text { features. Regulatory } \\
\text { costs offset based on } \\
\text { destination of each } \\
\text { call. Highly suitable } \\
\text { for VoIP conversion. }\end{array}$ \\
\hline
\end{tabular}

\section{POTS MODEL}

Earlier through example and plain old addition, division and multiplication, the POTS model was illustrated. It is perhaps one of the most reliable systems a company or consumer could have. It is also, many would argue, the most expensive model for a company with more than approximately 15 employees each needing a telephone on their desk.

The other mathematics that goes with the POTS model includes what is known as "access" charges. Access is the carrier services company way of stating that they are installing one or more physical POTS line(s) from your company's premise to the carrier service company's nearest building location.

The carrier company is going to assign each line a traditional telephone number and physically connect the line(s) at their end and provide you with dial tone on each line and the ability to make inbound and out bound calls.

For a business the access charge for a POTS line is approximately $\$ 65$ - $\$ 100$ per month per line. At this point you have basic POTS telephone service. If you want one or more of these lines to have features such as Caller ID or Conference (3-way) calling as discussed earlier, it would cost approximately $\$ 3$ and $\$ 2.75$ respectively per month per line. With the POTS Model all five regulatory categories apply to each and every POTS line in the business. Consequently, you can see that a small business with 15 lines can easily increase their TCO substantially if they are unaware of the finances of how the POTS Model works.

On the plus side, POTS lines are considered the highest voice quality and the standard by which industry measures quality in all other types of telephony systems. For today's consumer, POTS lines are quite a bargain since the carrier can usually provide broadband Internet for an additional small fee of approximately $\$ 25$ - \$35 per month.

The 911 service is the best available in the event of an emergency. POTS lines work when there are power outages. Also the carrier can usually offer residential customers various bundled packages that can save money. For example if the residential customer does a lot of interstate calling, they can get an interstate bundle for approximately $\$ 15$ that provides unlimited interstate calling at no extra charge. That usually means unlimited local calling and no recurring per-minute charges for out of state calls. On the corporate business side, it is entirely different in terms of how the carrier and company contract for bundled services.

\section{KTS MODEL}

The KTS is the abbreviated form of Key Telephony Station. All of the power of the system was invested 
into each and every telephone on this system. In the closet there was a KTS Switch chassis that merely connected all of the internal telephones via twistedpair lines to the internal side of the chassis in the closet.

The big savings came with how many external POTS lines were leased from the carrier company to be connected to the external side of the chassis. Access line costs are based on the algorithm that for every 68 employees, one POTS line is leased for the external side of the KTS chassis. If your company had 50 employees, they might order six to nine POTS lines from the carrier company. With the KTS Model, POTS lines from the carrier could be added and removed as required by the company.

Basically in the KTS Model all employees shared the same subset of POTS lines based on when they were calling and the duration of their calls. If all external POTS lines were occupied, the next inside caller or external caller would receive a busy signal. It became a function of the IT folks to monitor the calling patterns and to make sure the KTS System always had enough lines to handle the call volume of the business. In a correctly configured VoIP network, there would never be a busy signal because dedicated, channelized lines are used versus POTS lines. If the call volume was high in a VoIP network more channels of the dedicated line would be dynamically assigned to the current need with the telephony system.

\section{CENTREX}

CENTREX is the abbreviation for Central Exchange. In short, the customer company leased CENTREX lines on a monthly basis. Each line had an access cost and a usage cost. The usage cost was based on the recurring minutes of the line's use over the monthly billing cycle. If an employee on a particular line wanted "voice mail", this line would have an added charge of $\$ 10$ for this calling feature. As such all requested line call features were corresponded to line "add-on" charges. In addition, applicable regulatory charges per leased line were added to the total monthly cost of each line and then summarized into the monthly bill. With CENTREX everything was maintained by the Carrier services company. If voice mail needed to be added to a line, the carrier would take care of it at their end where the actual mainframe CENTREX Telephone System resided. If there was a problem with one of the lines that required a carrier company's technician to come out to the customer-company's site location, the time and materials for the site call are typically absorbed by the Carrier.

\section{PBX}

PBX is the abbreviation for Premise Business Exchange. It may also be referred to as Private Business Exchange. The more accurate term is the phrase with "Premise". Premise implies two things. One, the telephone system is physically located at the customer-company's site location. In the industry, this is understood to be "ON-PREM". Two, premise implies that the system is owned or leased by the customer-company versus the carrier services company. (As such one could call the PBX "private".) Under the CENTREX model, the mainframe CENTREX Telephone System residing at the carrier services company premise is essentially a large version of the PBX.

The cost of supporting the PBX system itself is considered high by comparison to the other three models. However, in larger companies with several hundred or even thousands of employees, the access, usage and regulatory cost savings from gaining the ability to use dedicated transport lines justifies the higher cost.

In addition, when you consider the alternatives of leasing individual POTS or CENTREX lines for every employee needing a telephone, along with the line access, usage, call line features and regulatory costs per month, the total MRC for the PBX model solution is justified by the businesses' TCO.

\section{CONCLUSION}

The PBX model is the most scalable telephony system model remaining out of the Final-Four Models. By enabling the use of dedicated transport lines, the company can not only grow their telephony system applications far more cheaply, they can integrate as well, computer data and videoconferencing applications. The PBX system can be upgraded with VoIP equipment and software interfaces that enable the company to begin its migration to a VoIP enabled enterprise.

The conversion to VoIP for any enterprise comes with high start-up costs. One way to reduce these start-up costs is to make use of existing systems, such as the PBX System and all of the telephone station units that would come with it.

For other companies that are currently using any of the other Final Four Models, keep in mind that a migration plan can be devised to phase in VoIP even while continuing business with the older model. In this way, a business does not have to lose current revenue while moving toward a full implementation of VoIP. 
Except in the case of the KTS Model, a company would not be losing any investments with their existing telephony systems. The POTS and CENTREX Models can be turned off (and back on again) at anytime, without any major loss of revenue or time.

With a KTS model, the systems chassis and telephone station units can be sold on the aftermarket once the conversion to VoIP is complete. Also the investment in the KTS Model generally runs between a few thousand dollars for the smaller systems up to six figures for the larger systems. If the company has one of the larger KTS systems, they come with interface ports to support VoIP. As a result, as with the PBX Model, a company running the larger KTS Systems can protect their investment and still convert to VoIP.

It has also been established that the conversion to VoIP is typically coupled with multi-month payback period for the total start-up costs. During these payback months and beyond the company is going to be enjoying the lower TCO resulting from the elimination of former telephony line access and usage charges.
The elimination of recurring line usage charges for all toll related calling. The company will now have more call features available to them than ever before. These features would include videoconferencing and all at no additional cost. Finally, the company would enjoy a reduction or elimination of the regulatory charges illustrated in Table $1[2 ; 3 ; 4 ; 6]$.

\section{REFERENCES}

6. Kelly, Timothy (2005). VoIP for Dummies, Wiley Publishing, Inc., Hoboken, New Jersey.

1. Audin, Gary. (2004). Architectures for Convergence. Business Communications Review. Oct., 4-8.

2. Blood, Steve; Johnson, Geoff; Lassman, Jay; (2008). Magic Quadrant for Corporate Telephony. Retrieved on April 26, 2008 from http://mediaproducts.gartner.com/reprints.

3. Bohlin, E., Brodin, K., Lundgren, A. and Thorngren, B. (2000). Convergence in Communications and Beyond. Elsevier.

4. COMPAEDIA (2009). Telecommunications Analysis for 2008. Retrieved on 4/24/09 from http://www.compaedia.com/Telecommunications

5. Kelly, Timothy V. (2006). Getting Switched. Retrieved on May 30, 2009 from http://searchunifiedcommunications.techtarget.co m/tip/0,289483,sid186_gci1130542,00.html. 